ELECTRONIC RESEARCH ANNOUNCEMENTS OF THE AMERICAN MATHEMATICAL SOCIETY

Volume 3, Pages 83-89 (August 28, 1997)

S $1079-6762(97) 00029-2$

\title{
ON POSITIVE ENTIRE SOLUTIONS TO THE YAMABE-TYPE PROBLEM ON THE HEISENBERG AND STRATIFIED GROUPS
}

\author{
GUOZHEN LU AND JUNCHENG WEI
}

(Communicated by Thomas Wolff)

\begin{abstract}
Let $\mathbb{G}$ be a nilpotent, stratified homogeneous group, and let $X_{1}$, $\ldots, X_{m}$ be left invariant vector fields generating the Lie algebra $\mathcal{G}$ associated to $\mathbb{G}$. The main goal of this paper is to study the Yamabe type equations associated with the sub-Laplacian $\triangle_{\mathbb{G}}=\sum_{k=1}^{m} X_{k}^{2}(x)$ on $\mathbb{G}$ :

$$
\triangle_{\mathbb{G}} u+K(x) u^{p}=0 .
$$

Especially, we will establish the existence, nonexistence and asymptotic behavior of positive solutions to (0.1). Our results include the Yamabe type problem on the Heisenberg group as a special case, which is of particular importance and interest and also appears to be new even in this case.
\end{abstract}

\section{InTRODUCTION}

In a series of papers [JM1], [JM2], [JM3] by D. Jerison and J. M. Lee, the Yamabe problem on CR manifolds was first studied, in conjunction with finding the best constant and extremal for the Sobolev inequality on the Heisenberg group. In particular, they studied the problem of conformally changing the contact form to one with constant Webster curvature in the compact setting. The Webster curvature is a scalar invariant of pseudo-Hermitian manifolds, which is defined independently by B. Webster $[\mathrm{W}]$ and N. Tanaka $[\mathrm{T}]$.

In this paper, we investigate the existence, nonexistence and asymptotic behavior of positive entire solutions to the following Yamabe-type problem associated with the sub-Laplacian $\triangle_{\mathbb{G}}=\sum_{k=1}^{m} X_{k}^{2}(x)$ on the stratified group $\mathbb{G}$ :

$$
\triangle_{\mathbb{G}} u+K(x) u^{\frac{Q+2}{Q-2}}=0,
$$

where $X_{1}, \ldots, X_{m}$ are left-invariant vector fields on $\mathbb{G}$, and $Q$ is the homogeneous dimension of $\mathbb{G}$ (see definition below), and $K(x)$ is locally Hölder continuous on $\mathbb{G}$ with respect to the Carnot metric as defined in [FS]. The results we obtain in this paper, of course, include the Heisenberg group $\mathbb{H}^{n}$ as a special case. These results are new even on $\mathbb{H}^{n}$.

Received by the editors June 12, 1997.

1991 Mathematics Subject Classification. Primary 35H05; Secondary 35J70.

Key words and phrases. Heisenberg group, stratified group, Yamabe problem, a priori estimates, asymptotic behavior, positive entire solutions.

The work of the first author was supported in part by the National Science Foundation Grant \#DMS96-22996.

The work of the second author was supported in part by an Earmarked Grant from RGC of Hong Kong.

(C) 1997 American Mathematical Society 
Equation (1.1) on the Heisenberg group $\mathbb{H}^{n}$ arises naturally in the study of the Cauchy-Riemann manifold $\left(\mathbb{H}^{n}, \theta\right)$ with contact form $\theta$, where $\mathbb{H}^{n}$ is a homogeneous group of real dimension $2 n+1$ with the underlying manifold

$$
\mathbb{H}^{n}=\mathbb{C}^{n} \times \mathbb{R}
$$

and whose group structure is given by

$$
(z, t) \cdot\left(z^{\prime}, t^{\prime}\right)=\left(z+z^{\prime}, t+t^{\prime}+2 \operatorname{Im}\left(z \cdot z^{\prime}\right)\right)
$$

for any two points $(z, t)$ and $\left(z^{\prime}, t^{\prime}\right)$ in $\mathbb{H}^{n}$.

The Lie algebra of $\mathbb{H}^{n}$ is generated by the left invariant vector fields

$$
X_{i}=\frac{\partial}{\partial x_{i}}+2 y_{i} \frac{\partial}{\partial t}, \quad Y_{i}=\frac{\partial}{\partial y_{i}}-2 x_{i} \frac{\partial}{\partial t}
$$

for $i=1, \ldots, n$. This is a prominent example of stratified Lie group of step two [FS]. The Kohn sub-Laplacian on $\mathbb{H}^{n}$ is defined as

$$
\triangle_{\mathbb{H}^{n}}=\sum_{i=1}^{n}\left(X_{i}^{2}+Y_{i}^{2}\right),
$$

which has received extensive attention for the past several decades.

The generators $\left\{X_{i}\right\}_{i=1}^{n}$ and $\left\{Y_{i}\right\}_{i=1}^{n}$ induce a canonical structure with a contact form $\theta$, which is determined up to a transformation

$$
\tilde{\theta}=u^{\frac{4}{Q-2}} \theta
$$

for some positive $u$. Each choice of $\theta$ corresponds to a pseudo-Hermitian structure on $\mathbb{H}^{n}$ and we denote

$$
\theta_{0}=2 \sum_{i=1}^{n}\left(x_{j} d y_{j}-y_{j} d x_{j}\right) .
$$

Given any contact form $\theta$ on $\mathbb{H}^{n}$, it gives rise to a Webster scalar curvature $R_{\theta}$. By the tranformation (1.3), the relationship between $R_{\theta}$ and $R_{\tilde{\theta}}$ is described as

$$
\frac{2 Q}{Q-2} \triangle_{\theta} u+R_{\theta} u=R_{\tilde{\theta}} u^{\frac{Q+2}{Q-2}},
$$

where $\triangle_{\theta}$ is the Laplacian on the pseudo-Hermitian manifold $\left(\mathbb{H}^{n}, \theta\right)$.

When we take $\theta=\theta_{0}, R_{\theta}$ becomes zero, and the equation (1.4) reduces to

$$
\frac{2 Q}{Q-2} \triangle_{\theta} u=R_{\tilde{\theta}} u^{\frac{Q+2}{Q-2}}
$$

under the transformation $\tilde{\theta}=u^{\frac{4}{Q-2}} \theta_{0}$.

Thus, equation (1.5) is the CR analogue of the Yamabe problem in the Euclidean setting.

The problem of finding a contact form $\tilde{\theta}$ on $\mathbb{H}^{n}$ with prescribed Webster scalar curvature $R_{\tilde{\theta}}$ is equivalent to finding a $C^{\infty}$ positive function $u$ on $\mathbb{H}^{n}$ such that $\tilde{\theta}=u^{\frac{4}{Q-2}} \theta_{0}$ and $u(x)$ satisfies the equation (1.5).

To state our theorems on the stratified group $\mathbb{G}$, we need to recall briefly some notions about groups. Let $\mathcal{G}$ be a finite-dimensional, stratified, nilpotent Lie algebra. Assume that

$$
\mathcal{G}=\bigoplus_{i=1}^{s} V_{i}
$$


and $\left[V_{i}, V_{j}\right] \subset V_{i+j}$ for $i+j \leq s,\left[V_{i}, V_{j}\right]=0$ for $i+j>s$. Let $X_{1}, \ldots, X_{m}$ be a basis for $V_{1}$ and suppose that $X_{1}, \ldots, X_{m}$ generate $\mathcal{G}$ as a Lie algebra. Thus we can choose a basis $\left\{X_{i j}\right\}$, for $1 \leq j \leq s, 1 \leq i \leq m_{j}$ for $V_{j}$, consisting of vectors of the form $X_{\alpha}$ for some multi-indices $\alpha$ of length $j$. In particular, $X_{i 1}=X_{i}, i=1, \ldots, m$ and $m=m_{1}$.

Let $\mathbb{G}$ be the simply connected Lie group associated to $\mathcal{G}$. Since the exponential mapping is a global diffeomorphism from $\mathcal{G}$ to $\mathbb{G}$, for each $g \in \mathbb{G}$, there is $x=$ $\left(x_{i j}\right) \in \mathbb{R}^{N}, 1 \leq i \leq m_{j}, 1 \leq j \leq s, N=\sum_{j=1}^{s} m_{j}$, such that

$$
g=\exp \left(\sum x_{i j} X_{i j}\right) .
$$

Thus we define a homogeneous norm function $|\cdot|$ on $\mathbb{G}$ by

$$
|g|=\left(\sum\left|x_{i j}\right|^{2 s ! / j}\right)^{1 / 2 s !} .
$$

On the Heisenberg group $\mathbb{H}^{n}$, for each $(z, t) \in \mathbb{H}^{n}$, we have

$$
|(z, t)|=\rho(z, t)=\left(|z|^{4}+t^{2}\right)^{\frac{1}{4}} .
$$

Let $\delta_{t}$ be a dilation on $\mathbb{G}$ defined by

$$
\delta_{t} x=\left(t^{j} x_{i j}\right)_{1 \leq i \leq m_{j}, 1 \leq j \leq s}
$$

for each $t>0$. It is easy to see that $\delta_{t}$ is an automorphism of $\mathbb{G}$ for each $t>0$. Lebesgue measure $d y$ is the bi-invariant Haar measure of $\mathbb{G}$ and the Jacobian of $\delta_{t}, J \delta_{t}$, is equal to $t^{Q}$, where $Q=\sum_{j=1}^{s} j m_{j}$ is called the homogeneous dimension of $\mathbb{G}$, which is usually greater than $\operatorname{dim} \mathbb{G}=N$. On the Heisenberg group $\mathbb{H}^{n}$, $Q=2 n+2$.

We mention in passing that some (existence and nonexistence) results, different from ours, on the Heisenberg group $\mathbb{H}^{n}$ were obtained by Garofalo and Lanconelli $[\mathrm{GL}]$ and Citti $[\mathrm{C}]$ on certain bounded or unbounded domains. We should also mention the earlier works by D. Jerison on solvability of the Dirichlet problem for a sub-Laplacian on the Heisenberg group [J1-2]. In the recent announcement ${ }^{1}$ of Brandolini, Rigoli, and Setti [BRS], they also considered the existence and nonexistence of positive entire solutions on the Heisenberg group and obtained some interesting results on $\mathbb{H}^{n}$. They appear to have used the method of comparison with radial equations in conjunction with the sub-super solution method. To be more precise, in [BRS] the authors consider, for nonnegative functions $a(x)$ and $b(x)$,

$$
\triangle_{\mathbb{H}^{n}} u+a(x) u-b(x)|u|^{\sigma-1} u=0, x=(z, t) .
$$

In the polar coordinates on $\mathbb{H}^{n}$, the sub-Laplacian can be written as

$$
\triangle_{\mathbb{H} n} u=\frac{|z|^{2}}{\rho(z, t)^{2}}\left(u^{\prime \prime}(\rho)+\frac{Q-1}{\rho} u^{\prime}(\rho)\right)
$$

Thus, the study of (1.6) will resemble the elliptic case (see the first general work due to W.-M. Ni [N] and many other subsequent works [CN], [LN], etc.). However, the presence of the density factor $\Psi(x)=\Psi(z, t)=\frac{|z|^{2}}{\rho(z, t)^{2}}$ on $\mathbb{H}^{n}$ in (1.7) makes

\footnotetext{
${ }^{1}$ We have not been able to obtain a copy of the full version of the paper containing the results announced in [BRS].
} 
the situation here more delicate, and one will need to assume that $a(x)$ and $b(x)$ satisfy

$$
a_{1}(|x|) \Psi(x) \leq a(x) \leq a_{2}(|x|) \Psi(x), \quad b_{1}(|x|) \Psi(x) \leq b(x) \leq b_{2}(|x|) \Psi(x),
$$

where $x=(z, t) \in \mathbb{H}^{n}$ and $|x|=\rho(z, t)$ (see [BRS]). Existence and nonexistence results of this type for positive entire solutions, obtained by using the comparison with the radial equations and also the method of sub-super solutions (as was done, e.g., in $[\mathrm{N}]$ by $\mathrm{Ni}$ ), were also independently derived on $\mathbb{H}^{n}$ by $\mathrm{Lu}$ in $[\mathrm{L}]$ for the equation

$$
\triangle_{\mathbb{H}^{n}} u+K(x) u^{p}=0 \text {, on } \mathbb{H}^{n} .
$$

Our methods in this paper use mainly an a priori estimate of the solutions, and thus avoid using the comparison with radial equations. As a result, we do not need to assume any condition on $K(x)$ involved with the degenerate factor $\Psi(x)$. Moreover, our method does not need the explicit structure of the Heisenberg group $\mathbb{H}^{n}$ and thus allows us to study the Yamabe type equation on a more general stratified, nilpotent group $\mathbb{G}$ :

$$
\triangle_{\mathbb{G}} u+K(x) u^{p}=0 .
$$

The theorems obtained in this paper consist of several parts. In Section 2, we obtain some existence results. In Theorems 2.1 and 2.2 there, we assume that $K(x)$ decays at infinity not more slowly than $|x|^{l}$ for some $l<-2$. These two theorems give existence results for all $1<p<\infty$. Theorem 2.3 deals with the case when $K(x)=\left(1+|x|^{2}\right)^{l / 2}$ for some $-2 \leq l<0$, and thus complements Theorems 2.1 and 2.2. We prove in this case the existence of positive solutions $u$ with $u \in L^{q}(\mathbb{G})$ for all $q \geq \frac{2 Q}{Q-2}$, while we restrict $p$ to the interval

$$
\frac{Q+2+2 l}{Q-2}<p<\frac{Q+2}{Q-2} \text {. }
$$

Section 3 of this paper contains the a priori estimates of positive solutions, which are stated as Theorems 3.1, 3.2 and 3.3. Section 4 deals exclusively with the Yamabe problem on the Heisenberg group $\mathbb{H}^{n}$. We derive the nonexistence result for positive

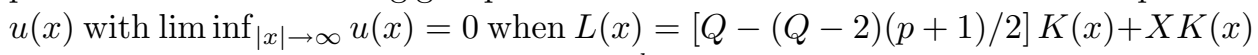
does not change signs and $|K(x)|=O\left(|x|^{l}\right)$ at infinity for some $l<-2$, where

$$
X K=\sum_{i=1}^{n}\left(x_{i} \frac{\partial K}{\partial x_{i}}+y_{i} \frac{\partial K}{\partial y_{i}}\right)+2 t \frac{\partial K}{\partial t} .
$$

This condition allows many candidates for $K$. We also give two other nonexistence results for any positive entire solutions when $K$ grows fast enough, or decays slowly; see Theorems 4.2 and 4.3 .

One word about the notation: throughout the paper, $C$ will generally denote an absolute constant which may vary from line to line; $K(x)$ is always assumed to be locally Hölder continuous.

\section{ACKNowledgement}

Both authors would like to thank Professor Wei-Ming Ni for his encouragement and his interest in this work. The first author would also like to thank him for helpful discussions. 


\section{EXISTENCE RESUlTS ON THE STRATIFIED GROUP}

Theorem 2.1. If $|K(x)| \leq C|x|^{l}$ for some $l<-2$ at infinity, then the equation (0.1) has infinitely many bounded positive solutions which are also bounded from below by positive constants.

Theorem 2.2. If $|K(x)| \leq C|x|^{l}$ for some $l<-2$ at infinity, then given any small positive number $\alpha$, there exists a bounded positive solution to equation (0.1) which satisfies $\lim _{|x| \rightarrow \infty} u(x)=\alpha$.

We now consider the eigenvalue problem

$$
\triangle_{\mathbb{G}} u=\lambda K(x) u^{p}, \quad x \in \mathbb{G}, \quad p>1
$$

and

$$
u \in C_{l o c}^{2}(G), \quad \lim _{|x| \rightarrow \infty} u(x)=0 .
$$

We have assumed here again that $K$ is a nonnegative, nontrivial and locally Hölder continuous function in $\mathbb{G}$, which satisfies

$$
|K(x)| \leq C|x|^{l} \text { at } \infty
$$

for some $-2 \leq l<0$.

Theorem 2.3. If we assume that $\frac{Q+2+2 l}{Q-2}<p<\frac{Q+2}{Q-2}$, then equation (2.2) has a positive solution pair $(\lambda, u)$ with $u \in L^{q}(\mathbb{G})$ for all $q \geq \frac{2 Q}{Q-2}$.

This theorem guarantees the existence of a positive solution of the Yamabe type equation because this $\lambda$ can be absorbed into the function $K(x)$.

\section{A PRIORI ESTIMATES ON THE STRATIFIED GROUP}

Theorem 3.1. Let $u$ be a bounded positive solution of (0.1) in $\mathbb{G}$, and $p>0$, with $\liminf |x| \rightarrow \infty u(x)=0$. Suppose that

$$
|K(x)| \leq C|x|^{l}
$$

at infinity for some $l<-2$. Then for any positive $\epsilon$ we have

$$
u(x) \leq \begin{cases}C|x|^{2-Q} \text { at } \infty & \text { if } p>\frac{Q+l}{Q-2}, \\ C_{\epsilon}|x|^{\frac{(1-\epsilon)(l+2)}{1-p}} \text { at } \infty & \text { if } p \leq \frac{Q+l}{Q-2},\end{cases}
$$

where $C_{\epsilon}$ only depends on $\epsilon$.

Theorem 3.2. Let $u$ be a bounded positive solution of (0.1) in $\mathbb{G}$, and $p>0$. Suppose that

$$
|K(x)| \leq C|x|^{l}
$$

at infinity for some $l<-2$. Then $u_{\infty}=\lim _{|x| \rightarrow \infty} u(x)$ exists. If we assume further that $K$ never changes sign in $\mathbb{G}$ and $|K(x)| \simeq|x|^{l}$ at infinity, then we have

$$
\left|u(x)-u_{\infty}\right| \simeq \begin{cases}C|x|^{2-Q} & \text { if } l<-Q, \\ C|x|^{2-Q} \log |x| & \text { if } l=-Q, \\ C|x|^{2+Q} & \text { if }-Q<l<-2,\end{cases}
$$

provided that $u_{\infty}>0$. 
Theorem 3.3. Let $u$ be a bounded positive solution of (0.1) in $\mathbb{G}$ for which $\liminf _{|x| \rightarrow \infty} u(x)=0$. Let $\alpha=\left(\alpha_{1}, \ldots, \alpha_{k}\right)$ be any multi-index. Suppose that $K(x)$ is smooth enough, say $K(x) \in C^{|\alpha|}(\mathbb{G})$ and

$$
|K(x)| \leq C|x|^{l}
$$

at infinity for some $l<-2$, and $p>\max \left\{0, \frac{Q+l}{Q-2}\right\}$. Then

$$
\left|X^{\alpha} u(x)\right| \leq C(1+|x|)^{|\alpha|-Q}, \text { for all } x \text { in } \mathbb{G},
$$

where $X^{\alpha}=X_{i_{1}}^{\alpha_{1}} \cdots X_{i_{k}}^{\alpha_{k}}, 1 \leq i_{j} \leq m,|\alpha|=\alpha_{1}+\alpha_{2}+\cdots+\alpha_{k}$, and $C$ is a positive constant.

\section{Nonexistence Results on the Heisenberg Group}

All the results stated in the previous sections apply as well to the Heisenberg group. However, by taking advantage of the special structure of $\mathbb{H}^{n}$, we can do more precise analysis for (1.1). We now state a nonexistence theorem on the Heisenberg group $\mathbb{H}^{n}$. We denote

$$
X K=\sum_{i=1}^{n}\left(x_{i} \frac{\partial K}{\partial x_{i}}+y_{i} \frac{\partial K}{\partial y_{i}}\right)+2 t \frac{\partial K}{\partial t} .
$$

Theorem 4.1. Suppose that $|K(x)| \leq C|x|^{l}$ at infinity for some $l<-2, K \in$ $C^{1}\left(\mathbb{H}^{n}\right)$, and the function $L(x)=[Q-(Q-2)(p+1) / 2] K(x)+X K(x)$ never changes sign in $H^{n}$. Then equation (1.1) does not possess any positive bounded solution $u$ satisfying $\liminf |x| \rightarrow \infty u=0$.

To state the next two theorems, we need to introduce some notation on the Heisenberg group $\mathbb{H}^{n}$. Let

$$
B_{r}(x)=B(x, r)=\left\{y \in \mathbb{H}^{n}: \rho(x, y)=\left|x \cdot y^{-1}\right|<r\right\}
$$

be the Heisenberg ball centered at $x$ and with radius $r>0$, and

$$
\partial B_{r}(x)=\left\{y \in \mathbb{H}^{n}: \rho(x, y)=r\right\} .
$$

Given any nonzero function $K$ we define

$$
\bar{K}(r)=\left(\frac{1}{\left|\partial B_{r}(0)\right|} \int_{\partial B_{r}(0)} \frac{\Psi(z, t)}{K(z, t)^{\frac{Q-2}{4}}} \frac{d \sigma}{|\nabla \rho|}\right)^{-\frac{4}{Q-2}}
$$

where $d \sigma$ is the surface measure on $\partial B_{r}(0)$, and $|\nabla \rho|$ is the classical gradient of the distance function $\rho(z, t), \Psi(z, t)=\frac{|z|^{2}}{\rho(z, t)^{2}}$.

If $K(x)=K(z, t)=K(|(z, t)|)=K(\rho(z, t))$, then an easy calculation shows that

$$
\bar{K}(\rho)=C_{Q} K(\rho),
$$

with $C_{Q}$ an absolute constant.

Theorem 4.2. If $K(x) \geq 0$ in $\mathbb{H}^{n}$, and $\bar{K}(\rho) \geq C \rho^{p}$ at infinity for some constants $C>0$ and $p>2$, then the equation

$$
\triangle_{\mathbb{H}^{n}} u+K(x) \Psi u^{\frac{Q+2}{Q-2}}=0, \text { on } \mathbb{H}^{n}
$$

does not possess any positive entire solutions in $\mathbb{H}^{n}$. 
Theorem 4.3. If $K(x) \geq 0$ in $\mathbb{H}^{n}$, and $\bar{K}(\rho) \geq \frac{C}{\rho^{k}}$ at infinity for some constants $C>0$ and $k<2$, then the equation

$$
\triangle_{\mathbb{H}^{n}} u=K(x) \Psi u^{\frac{Q+2}{Q-2}} \text {, on } \mathbb{H}^{n}
$$

does not possess any positive entire solutions in $\mathbb{H}^{n}$.

Remarks. By the observation (4.1), the $\bar{K}(\rho)$ can be replaced by $K(\rho)$ for radial $K$ in the statements of the above two theorems. We can extend as well Theorems 4.2 and 4.3 to the case where the critical exponent $\frac{Q+2}{Q-2}$ is replaced by any $p>1$, with the appropriate growth or decay conditions on $K$.

\section{REFERENCES}

[BRS] L. Brandolini, M. Rigoli, and A. G. Setti, On the existence of positive solutions of Yamabe-type equations on the Heisenberg group, Electronic research announcements of the AMS, Dec. 1996, 2 (1996), 101-107.

[C] G. Citti, Semilinear Dirichlet problem involving critical exponenent for the Kohn Laplacian, Annali di Mate. Pura Appl. 169 (1995), 375-392. MR 96j:35071

[CN] K. S. Cheng and W.-M. Ni, On the structure of the conformal scalar curvature equation on $\mathbb{R}^{n}$, Indiana Univ. Math. J. 41 (1992), 261-278. MR 93g:35040

[FS] G. B. Folland and E. M. Stein, Hardy spaces on homogeneous groups, Princeton Univ. Press, 1982. MR 84h: 43027

[GL] N. Garofalo and E. Lanconelli, Existence and nonexistence results for semilinear equations on the Heisenberg group, Indiana Univ. Math. J. 41 (1992), 71-98. MR 93h:35071

[H] L. Hörmander, Hypoelliptic second order differential equations, Acta Math. 119 (1967), 147-171. MR 36:5526

[J1-2] D. Jerison, The Dirichlet problem for the Kohn Laplacian on the Heisenberg group, I, II, J. Functional Anal. 43 (1981), 97-142, 224-257. MR 83c:58081a; MR 83c:58081b

[JM1] D. Jerison and J. M. Lee, The Yamabe problem on CR manifolds, J. Diff. Geom. 25 (1987), 167-197. MR 88i:58162

[JM2] D. Jerison and J. M. Lee, Intrinsic CR normal coordinates and the CR Yamabe problem, J. Diff. Geom. 29 (1989), 303-343. MR 90h:58083

[JM3] D. Jerison and J. M. Lee, Extremals for the Sobolev inequality on the Heisenberg group and the CR Yamabe problem, J. Amer. Math. Soc. 1 (1988), 1-13. MR 89b:53063

[LN] Y. Li and W.-M. Ni, On conformal scalar curvature equations in $\mathbb{R}^{n}$, Duke Math. Journal, 57 (1988), 895-924. MR 90a:58187

[L] G. Lu, unpublished manuscript, 1994.

[N] W.-M. Ni, On the elliptic equation $\triangle u+K(x) u^{\frac{n+2}{n-2}}=0$, its generalization and applications in geometry, Indiana Univ. Math. J. 31 (1982), 493-529. MR 84e:35049

[T] N. Tanaka, A differential geometric study on strongly pseudo-convex manifolds, Kinokuniya, Tokyo, 1975. MR 53:3361

[W] S. M. Webster, Pseudo-Hermitian structures on a real hypersurface, J. Diff. Geom. 13 (1978), 25-41. MR 80e:32015 45435

Department of Mathematics and Statistics, Wright State University, Dayton, OH

E-mail address: gzlu@math.wright.edu

Department of Mathematics, Chinese University of Hong Kong, Shatin, N.T., Hong KONG

E-mail address: wei@math.cuhk.edu.hk 